(C) 20044 American Chemical Society, J. Phys. Chem. B, Chen jp031077n Supporting Info Page 1 J

\title{
Silver Nanoplates: Size Control in 2-Dimensions and Formation Mechanisms
}

\author{
Sihai Chen, ${ }^{\dagger}$ and David L. Carroll, ${ }^{\ddagger}$ \\ ${ }^{\dagger}$ School of Materials Science and Engineering, Clemson University, Clemson, South Carolina 29634-0971, and ${ }^{\ddagger}$ Center for \\ Nanotechnology, Department of Physics, Wake Forest University, Winston-Salem, North Carolina 27109-7507
}

\section{Supporting information:}

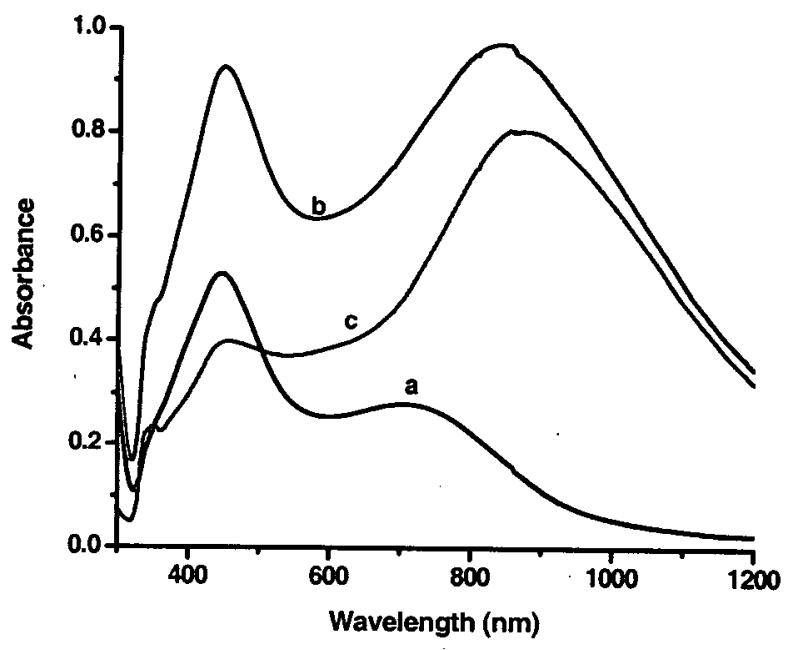

Figure S1. In order to clarify the origin of the absorption peak around $415-442 \mathrm{~nm}$, separation experiments can be carried out by centrifugation. Here, since we found that the large nanoplates can easily precipitate out even by placing in the ambient condition, we have aged the nanoplate solutions of samples $b$ in Figure 1 for six months. The absorption spectra were (a) supernatant solution, and (b) solution after shaking to be homogeneous, and (c) difference spectrum between (b) and (a). The spectrum (a) of the supernatant solution gives an apparent peak at $450 \mathrm{~nm}$ and a shoulder peak at around $715 \mathrm{~nm}$, indicating that the solution contains spherical and smaller sized nanoplates. The spectrum (c) of larger nanoplates shows three peaks at 350, 450 and $875 \mathrm{~nm}$, with their intensity ratios reasonably consistent with the theoretical prediction. ${ }^{\text {S1.S2 }}$. Also noticed is a blue-shift of about $150 \mathrm{~nm}$ for the in-plane dipole plasmon band of the aged solution (spectrum b) comparing to the not aged one, probably due to the rounding effect of the nanoplates as we observed before. ${ }^{\mathrm{s} 3}$ 


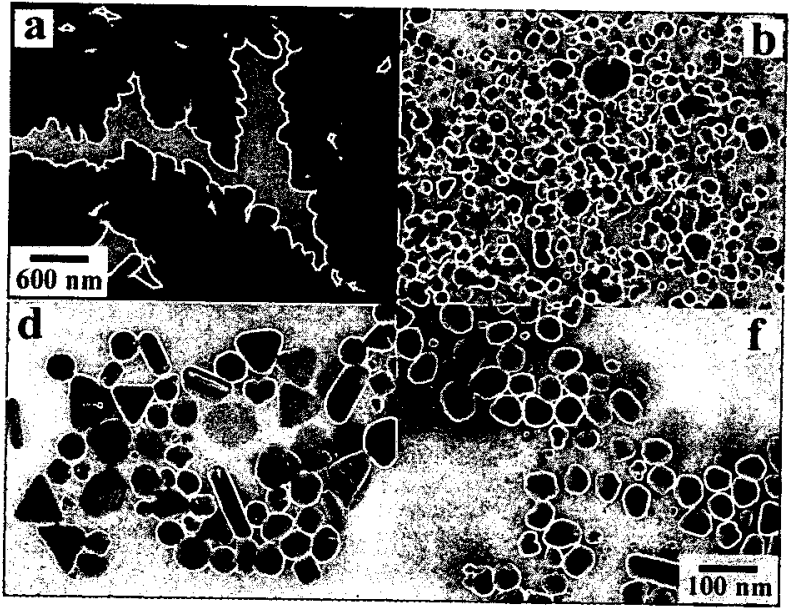

Figure S2. TEM images of the silver particles obtained at different CTAB concentrations. The [CTAB]/[Ag] molar ratio are (a) 0, (b) 1 , (d) 30, and (f) 200. Other conditions are the same as that in Figure 6. The scale bar in the TEM photos in (f) also applies to (b) and (d).
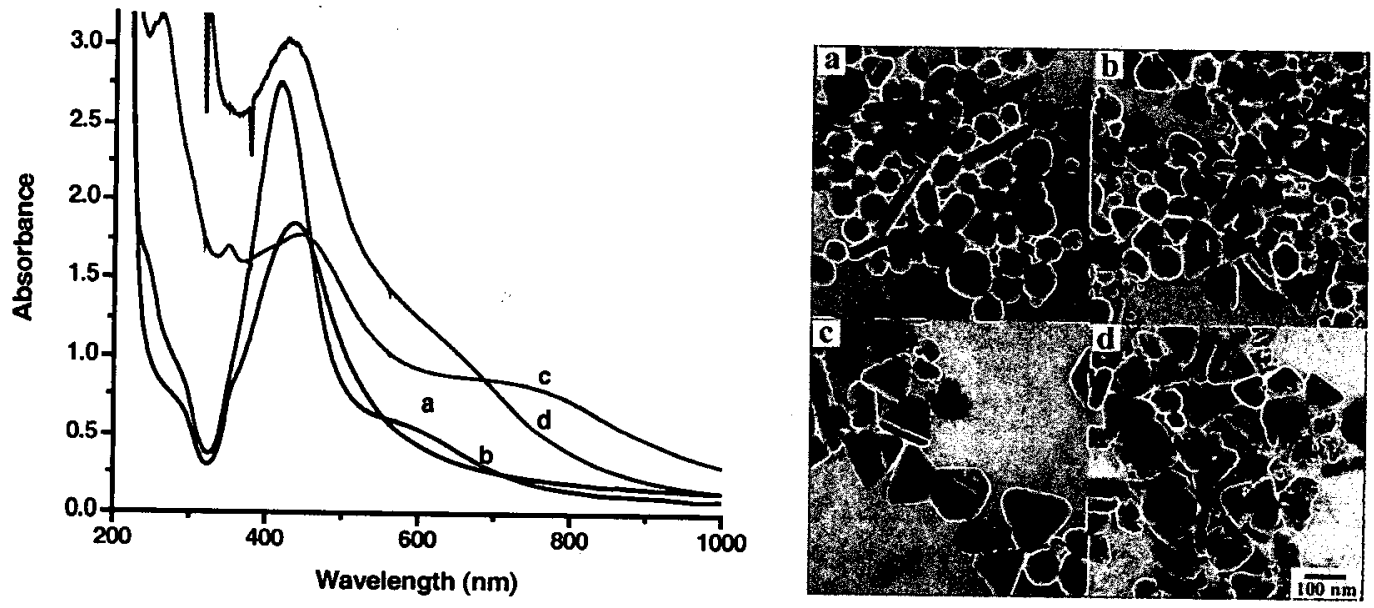

Figure S3. Absorption spectra and TEM images of the silver particles obtained under standard condition using different reducing agent (final $\mathrm{pH}$ of the solution shown in parenthesis): (a) sodium L-ascorbate (11.7), (b) L-ascorbic acid 6-palmitate (11.7), (c) hydroquinone (11.9), (d) 4-(methylamino) phenol sulfate (12.4), and (e) hydroxylamine hydrochloride (12.5). The scale bar applies to all images. In all cases, plates are formed.

(S1) Hao, E.; Kelly, K. L.; Hupp, J. T.; Schatz, G. C. J. Am. Chem. Soc. 2002, 124, 15182

(S2) Jin, R. C.; Cao, Y. W.; Mirkin, C. A.; Kelly, K. L.; Schatz, G. C.; Zheng, J. G. Science 2001, 294, 1901.

(S3) Chen, S.; Fan, Z.; Carroll, D. L. J. Phys. Chem. B 2002, 106, 10777. 